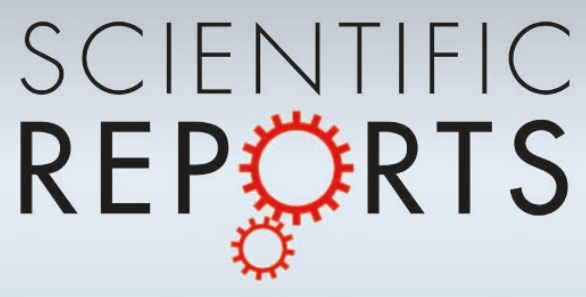

OPEN

SUBJECT AREAS:

FLUID DYNAMICS

MECHANICAL ENGINEERING

Received

9 June 2014

Accepted

4 November 2014

Published

10 December 2014

Correspondence and requests for materials should be addressed to N.A.P. (n-patankar@ northwestern.edu) or M.A.M. (maciver@ northwestern.edu)

* These authors contributed equally to this work.

\section{Separability of drag and thrust in undulatory animals and machines}

\author{
Rahul Bale ${ }^{*}$, Anup A. Shirgaonkar * , Izaak D. Neveln ${ }^{2}$, Amneet Pal Singh Bhalla' ${ }^{1}$ Malcolm A. Maclver ${ }^{1,2,3}$ \\ \& Neelesh A. Patankar ${ }^{1}$
}

\begin{abstract}
'Department of Mechanical Engineering, Northwestern University, ${ }^{2}$ Department of Biomedical Engineering, Northwestern University, ${ }^{3}$ Department of Neurobiology, Northwestern University, 2145 Sheridan Road, Evanston, IL 60208, USA.
\end{abstract}

For nearly a century, researchers have tried to understand the swimming of aquatic animals in terms of a balance between the forward thrust from swimming movements and drag on the body. Prior approaches have failed to provide a separation of these two forces for undulatory swimmers such as lamprey and eels, where most parts of the body are simultaneously generating drag and thrust. We nonetheless show that this separation is possible, and delineate its fundamental basis in undulatory swimmers. Our approach unifies a vast diversity of undulatory aquatic animals (anguilliform, sub-carangiform, gymnotiform, bal-istiform, rajiform) and provides design principles for highly agile bioinspired underwater vehicles. This approach has practical utility within biology as well as engineering. It is a predictive tool for use in understanding the role of the mechanics of movement in the evolutionary emergence of morphological features relating to locomotion. For example, we demonstrate that the drag-thrust separation framework helps to predict the observed height of the ribbon fin of electric knifefish, a diverse group of neotropical fish which are an important model system in sensory neurobiology. We also show how drag-thrust separation leads to models that can predict the swimming velocity of an organism or a robotic vehicle.

T he hydrodynamics of aquatic locomotion has importance across multiple domains, from basic biology to the engineering of highly maneuverable underwater vehicles. Within biology, beyond the interest in aquatic locomotion, there is extensive use of several aquatic model systems within neuroscience such as lamprey for research into spinal cord function ${ }^{1}$, zebrafish for developmental neuroscience ${ }^{2}$, and weakly electric fish for the neurobiology of sensory processing (reviews: refs. 3,4 ). Within engineering, the maneuverability and efficiency of fish is inspiring new styles of propulsion and maneuvering in underwater vehicles ${ }^{5-7}$. The implementation of engineered solutions will depend on the resolution of open issues in hydrodynamics of aquatic locomotion. Finally, an understanding of the hydrodynamics of aquatic locomotion is critical for insight into the evolution of fish $^{8}$ and their land-based descendants.

While the hydrodynamics of swimming organisms have been studied actively for almost a century, there are key questions that remain unresolved. This work focuses on one such unresolved issue that pertains to the mechanisms of drag and thrust generation. The decomposition of the total force on a swimming organism into drag and thrust is desirable because it can fundamentally reveal how an organism produces forward push to balance the resistance to motion from the surrounding fluid. It can also lead to simple quantitative models to predict swimming velocity of organisms or artificial underwater vehicles based on their kinematics.

If we consider a boat with a propeller, the decomposition of thrust and drag is straightforward since all of the thrust is coming from the propeller, and most of the drag is coming from the hull. However, for swimmers that use undulatory motions for propulsion (Fig. 1), drag-thrust decomposition is not straightforward. For example, in the case of anguilliform swimmers such as eels, where the entire body undulates, there are no distinct portions of the body that alone produce thrust or cause drag. In other groups of fishes there are undulatory elongated fins along the ventral midline (Fig. 2a, knifefish such as those of the Gymnotiformes and Notopteridae), dorsal midline (Gymarchus nilotics, the oarfish Regalecus glesne), along ventral and dorsal midlines (triggerfish of the Balastidae), and along the lateral margins of the body (certain rays and skates of the Batoidea, cuttlefish). For these animals, undulating fins may be regarded as the primary thrust generators and the relatively straight body may be regarded as the primary source of drag. However, this apparent decomposition of drag and thrust regions should not be considered to imply that an undulatory fin itself has no drag. This is clearly not the case because a hypothetical undulatory ribbon fin that is not attached to a body will undergo steady swimming. The drag of the ribbon fin and the thrust it generates will be in balance in that case. 

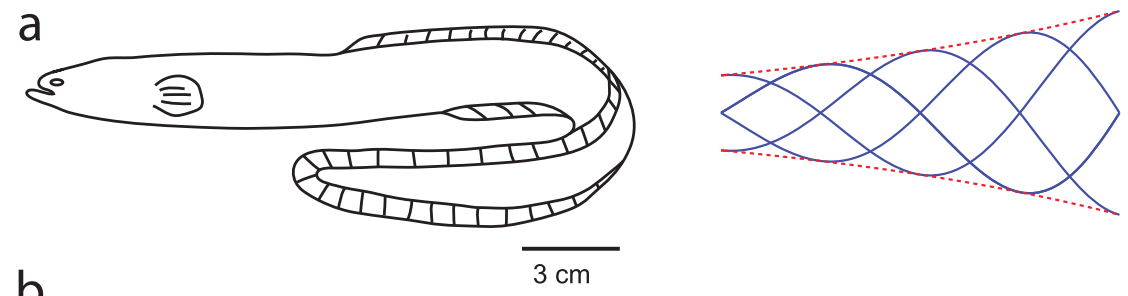

$\mathrm{b}$
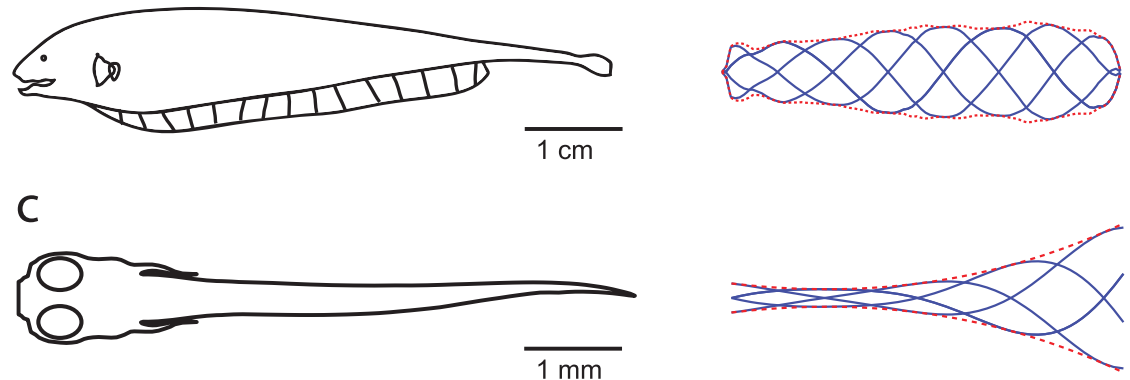

Figure $1 \mid$ A sampling of swimmers that use undulatory motions for propulsion, along with multiple snapshots of the body midline (a and c) or fin (b) shape during swimming. (a) An anguilliform swimmer, such as an american eel. (b) A gymnotiform swimmer, such as the black ghost knifefish.

(c) A sub-carangiform swimmer, such as a zebrafish larva. The red dotted line along the body and fin snapshots indicates the envelope across multiple undulations. In this work we show that across these kinematics, the total force on the propulsor can be decomposed into drag and thrust in spite of these forces being intermingled on the propulsor. The figures were drawn in Adobe Illustrator by I.D.N. and M.A.M.

Arguably the most widely cited analysis of drag-thrust decomposition is due to Lighthill'. He considered the force generated from undulatory motion by elongated animals such as eels ${ }^{10}$, and later, with Blake, considered forces on the ribbon fins of balistiform and gymnotiform swimmers ${ }^{11}$. The analysis was based on a "reactive" theory of propulsion that was proposed for high Reynolds number swimming. Lighthill considered a decomposition of the force on the body into resistive and reactive components that lead to drag and thrust, respectively. He defined the force on a section of the body as resistive if it depends, linearly or non-linearly, on the instantaneous velocity of that section relative to the surrounding fluid. Reactive forces were defined as those due to inertia of the surrounding fluid (the "added mass" effect), proportional to the rate of change of the relative velocity between the fluid and surface of the swimming body. Lighthill then provided expressions for the reactive thrust force using a simplified potential flow theory ${ }^{10,11}$. No model for drag was developed.

To address this long-standing issue, we define three fundamental principles for the decomposition of forces arising from undulatory swimming into drag and thrust. First, (D1) the body movement (kinematics) creating drag needs to be separated from the body movement creating thrust, such that the sum of these two movements results in the originally observed swimming motion of the animal. Second, (D2) these decomposed movements should be such that the surface of the body will move in a continuous fashion so that the kinematics can be realized in experiments or simulations. Third, (D3) the sum of the force due to the drag-inducing movement with the force due to the thrust-inducing movement needs to equal the force estimated from the original (undecomposed) movement of the fish. Requirement (D2) will enable three independent experiments to estimate forces and develop predictive models for swimming: i) the force based on the undecomposed motion; ii) the force resulting purely from the drag kinematics, defined as drag; iii) the force resulting purely from the thrust kinematics, defined as thrust.

In this work we propose a new way to decompose drag and thrust that satisfies conditions D1 to D3. Using an idealized elongated anal fin (hereafter ribbon fin) of weakly electric fish (Fig. 2a) but with no body as a model system, we present results from simulations and experiments supporting our approach. The scope of applicability of the decomposition will be further demonstrated through simulations of the eel Anguilla rostrata, the larval zebrafish Danio rerio, the black ghost knifefish Apteronotus albifrons, and the mackerel Scomber scombrus. These examples show where the decomposition is valid (which includes the cases shown in Fig. 1), and where it becomes invalid. For a case where it is valid-swimming with elongated fins such as in the knifefish-we go on to show that drag-thrust decomposition can be used to predict an important morphological feature: the height of the fin that minimizes the cost of transport, a measure of the energy needed for locomotion per unit distance. Our predictions agree well with the measured height of the fin in a sample of 13 species in a representative family of knifefishes, the Apteronotidae.

\section{Results}

Kinematic decomposition. To demonstrate drag-thrust decomposition, we consider the same model problem considered by Lighthill and Blake ${ }^{11}$ and analyze the forces on the elongated median fin (hereafter "ribbon fin") of a gymnotiform swimmer (Fig. 2a). We numerically simulate a translating ribbon fin with a traveling wave (Fig. 2b) along it. The traveling wave on the ribbon fin was described by the angular position of any point on the ribbon fin $\theta(x, t)=\theta_{\max } \sin 2 \pi\left(\frac{x}{\lambda}-f t\right)$, where $\theta_{\max }$ is the maximum angle of excursion, $x$ is the coordinate in the axial direction, $f$ is the frequency, and $\lambda$ is the wavelength of undulations. In the simulations there is no attached body. The fin morphology is shown in Fig. 2c. The sign convention for velocity and force is described in the Methods. The force on the ribbon fin from the fluid is numerically computed for different values of the translational velocity $U$ of the fin and the traveling wave velocity $U_{\mathrm{w}}$ (given by $f \lambda$ ). The wave motion is caused by the lateral oscillatory velocity field $V_{\mathrm{w}}$ on the fin surface. $V_{\mathrm{w}}$, and by consequence $U_{\mathrm{w}}$, were varied by changing the frequency $f$ of the traveling wave. The fin had two undulations along its length ${ }^{12,13}$.

To understand our proposed kinematic decomposition, consider a generic waveform of constant amplitude that has a lateral oscillatory velocity $V_{\mathrm{w}}$ and a corresponding traveling wave velocity $U_{\mathrm{w}}$. Let $U$ be the forward velocity of the fin as a whole. This is the undecomposed kinematics (Fig. 3 left bottom panel) which is decomposed into two parts (Fig. 3 left top two panels). The first decomposed motion, which we term the drag-causing perfect slithering motion, occurs when the forward velocity of the fin is equal to the backward velocity of the 

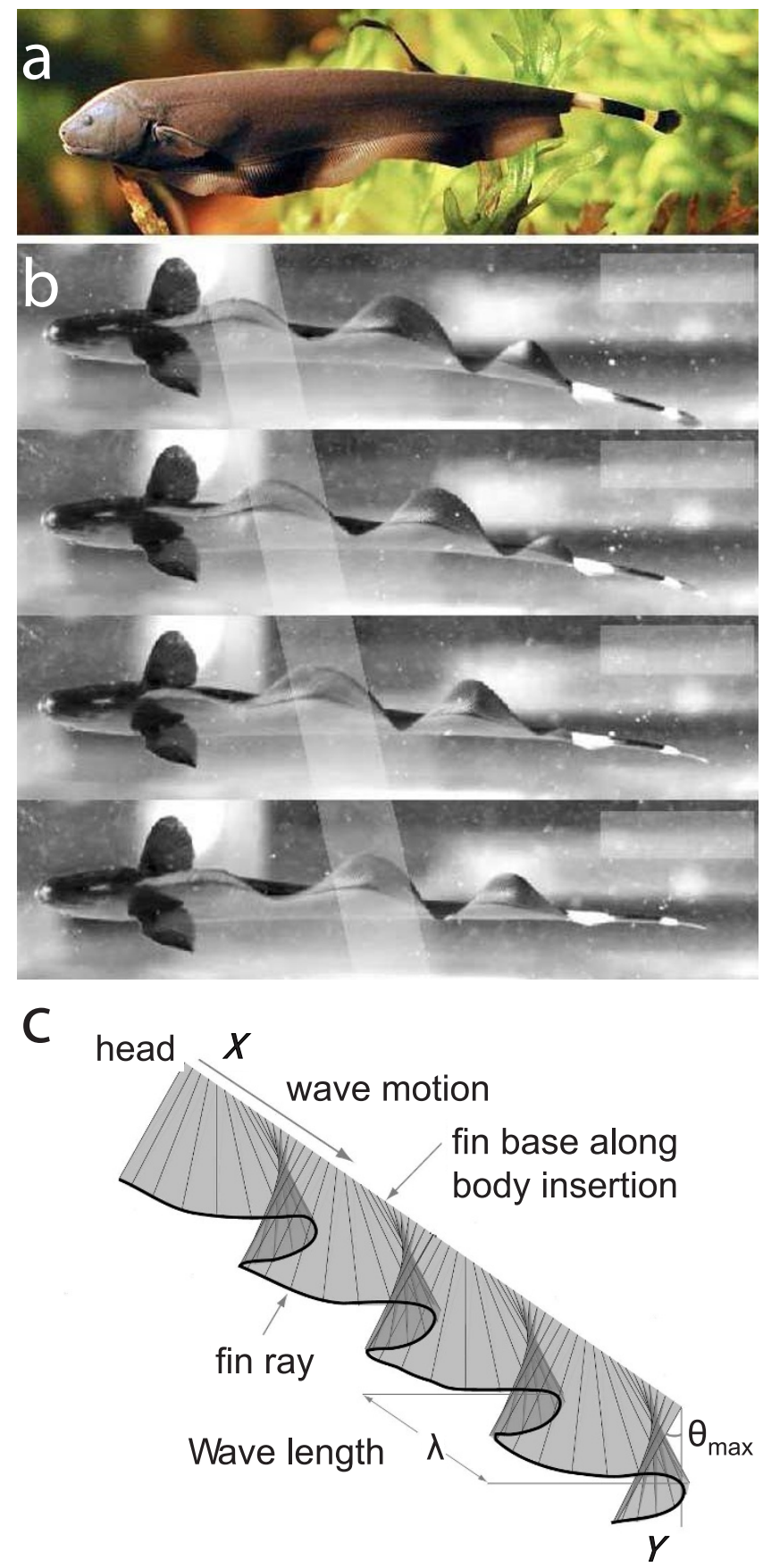

Figure 2 (a) A median fin (ribbon fin) undulatory swimmer (gymnotiform swimmer): Apteronotus albifrons, the black ghost knifefish of South America. (b) A backward traveling wave on the ribbon fin. (c) Geometric configuration of the ribbon fin without the body for computations. Figure adapted from Fig. 1 of ref. 13, reproduced with permission from M.A.M (M.A.M owns the copyrights to all figures/images in ref. 13).

traveling wave $\left(U_{\mathrm{w}}\right)$. As a result, the fin appears to move along a stationary sinusoidal track (Fig. 3 left top panel). Each point on the fin has a velocity that is tangential to its surface (Fig. 3 left top panel). In this case the fluid is dragged forward by the tangential velocity along the fin surface which results in a backward (drag) force on the fin from the fluid. The second decomposed motion, which we term the thrust generating frozen fin motion, occurs when the fin is frozen in its undulatory shape, and that frozen shape drifts backward with a velocity equal to the velocity of the traveling wave minus the translational velocity of the fin $\left(U_{\mathrm{w}}-U\right)$, when $U_{\mathrm{w}}$ is greater than $U$. This motion pushes the fluid backward which in turn produces a forward (thrust) force on the fin (Fig. 3 left middle panel). In the idealization of the traveling wave as a sinusoid of constant amplitude, superposition of the kinematics of the perfect slithering and frozen fin motions results in the undecomposed kinematics of the fin surface (Fig. 3 left bottom panel). This fulfills both condition D1 (separated body movements add up to give original body movements) and condition D2 (the separated body movements are without discontinuities and physically realizable).

The above kinematic decomposition would be exact in case of an infinitely long fin. However, this is not the case for the finite fin length that we consider. For example, in the frozen fin case, the wave can be considered frozen in different phases. Despite this, our computational fluid simulations show that the thrust force does not depend strongly on the phase as long as there is more than one full wave on the fin, as is the case here. We next show that the kinematic decomposition also fulfills the requirement that the force of drag and the force of thrust sum to the undecomposed force from the fin (D3).

Dynamic decomposition. Dynamic decomposition here implies the decomposition of forces on the swimming body. Without loss of generality we consider a traveling wave that is moving backward, as in Fig. 2b. If the decomposition into drag and thrust is valid, then the total force $F$ on the fin should satisfy the following equation

$$
F\left[U, U_{\mathrm{w}}\right]=\operatorname{sgn}\left(U_{\mathrm{w}}-U\right) T\left[U_{\mathrm{w}}-U\right]-D\left[U_{\mathrm{w}}\right],
$$

where square brackets indicate "function-of", $T$ is thrust, $D$ is drag, and $\operatorname{sgn}[\cdot]$ gives the sign of the argument. The data for $F\left[U, U_{\mathrm{w}}\right]$ are plotted in Fig. 4a1-a4. We performed a separate set of simulations for the perfect slithering motion $\left(U_{\mathrm{s}}=U_{\mathrm{w}}\right)$ for different values of $U_{\mathrm{w}}$. The force on the ribbon-fin in this case is $D\left[U_{\mathrm{w}}\right]$, which is plotted in Fig. $4 b$.

Using Eqn. 1 and the results in Fig. $4 \mathrm{~b}$ we calculate the thrust $T\left[U_{\mathrm{w}}\right.$ $-U]$ for each data point in Fig. 4a1-a4. If the decomposition is valid then the resulting data should be a well defined function of $U_{w}-U$. This is found to be so in Fig. 4c. Additionally, the results for $T\left[U_{\mathrm{w}^{-}}\right.$ $U]$, obtained above, should also match results from another set of simulations for the frozen fin case. To check this we performed frozen fin simulations for different values of the fin translational velocity $U_{\mathrm{f}}$. There is no traveling wave in this case. The force on the ribbon fin in this case is $T\left[U_{\mathrm{f}}\right]$ which should be the same function as $T\left[U_{\mathrm{w}}-U\right]$ with $U_{\mathrm{f}}$ replaced by $\left(U_{\mathrm{w}}-U\right)$. The solid line of Fig. $4 \mathrm{c}$ confirms this expectation.

Thus, we have two new results: first, an approach to separate the mechanisms of drag and thrust, and second we obtain a correlation not only for the thrust (Fig. 4c) but also for the drag (Fig. 4b) on an undulatory propulsor.

The spatial segregation of drag-and thrust-related flows. Consider a ribbon fin moving with $U=3 \mathrm{~cm} / \mathrm{s}$ and $U_{\mathrm{w}}=15 \mathrm{~cm} / \mathrm{s}$. Through simulation, the total forward force is found to be $0.46 \mathrm{mN}$. The drag causing perfect slithering mode has $U=15 \mathrm{~cm} / \mathrm{s}$ and $U_{\mathrm{w}}=15 \mathrm{~cm} / \mathrm{s}$. The thrust generating frozen mode has no wave velocity but has a backward velocity of $U_{\mathrm{w}}-U=12 \mathrm{~cm} / \mathrm{s}$. Separate simulations were conducted for the drag and thrust causing modes. The calculated thrust and drag forces were $0.92 \mathrm{mN}$ and $0.52 \mathrm{mN}$, respectively. The difference is $0.4 \mathrm{mN}$ which is close to $0.46 \mathrm{mN}$ computed for the un-decomposed case, i.e., Eqn. 1 is approximately satisfied. We plot the simulated axial velocity and pressure for these three cases in Fig. 5 for the same phase of the fin. The slithering drag mode has thin boundary layers outside of which the velocity has low magnitude and does not have strong spatial gradients. On the other hand, the frozen thrust mode has strongly separated regions behind the troughs and crests of the wave along the fin. This velocity field and its gradients are significant outside the boundary layer region of the slithering mode. Wherever the velocity due to one mode is high, the velocity 


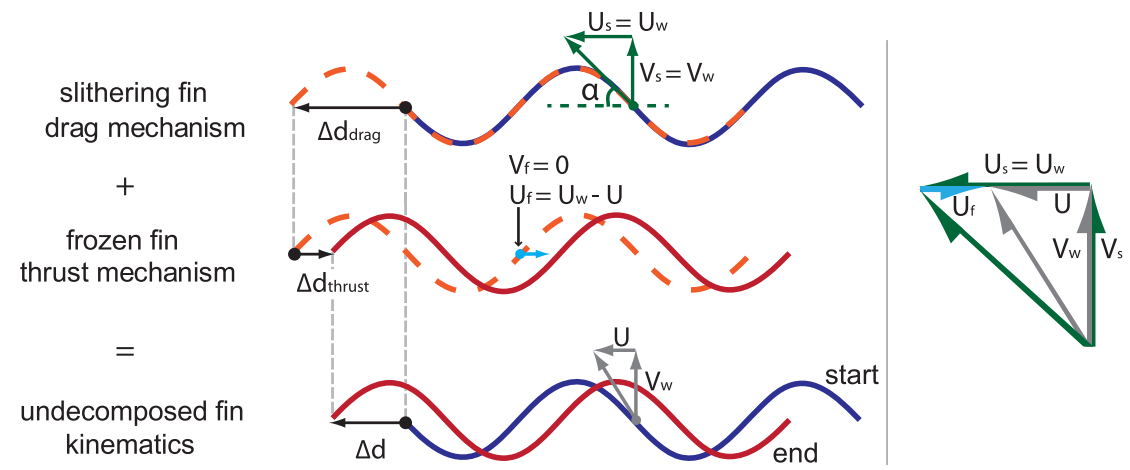

Figure 3 The proposed kinematic decomposition into drag and thrust producing mechanisms. The top two rows split the decomposed kinematics into two successive movements. First, in the slithering fin and drag producing mechanism, the fin follows a sinusoidal track. Therefore, the forward speed $U_{\mathrm{s}}=$ $U_{\mathrm{w}}$, which is the backward speed of the traveling wave. Also, the lateral velocity field is equal to that of the undecomposed fin kinematics. Over a time span equal to half a period, the fin moves from the solid blue configuration to the dotted orange configuration, with a longitudinal displacement, $\Delta d_{\mathrm{drag}}$. The frozen fin and thrust producing mechanism has no lateral velocity field and moves backward at a speed equal to $U_{\mathrm{w}}-U$, where $U$ is the speed of the undecomposed fin kinematics. Therefore, over the same time span, the frozen fin moves backward with a longitudinal displacement, $\Delta d_{\text {thrust }}$ from the dotted orange line to the solid red line. The slithering and frozen fin kinematics add to the undecomposed fin kinematics, where the blue and red lines indicate the start and end configurations, respectively. The velocity vectors are redrawn on the right to show how the velocity vectors of the frozen and slithering fins add to equal those of the undecomposed fin kinematics. The sign convention for velocity and force is described in the Methods.

due to the other mode is low. The coupling of the drag and thrust causing modes, through the nonlinear inertia term $((u \cdot \nabla) u)$ in the Navier-Stokes equations (Eqn. S11 in SI), is weak. Furthermore, Fig. 5 shows that the dominant pressure regions due to the two modes are also spatially segregated. The low pressure due to the thrust-causing frozen fin mode is dominant behind the wave troughs and crests, whereas the low pressure due to the slithering mode is dominant away from the separation region in the concave part of the wave shape. This spatial segregation of the drag- and thrust-related flows is the fundamental basis of the success of the drag-thrust decomposition. Linear behavior of the nonlinear Navier-Stokes equations has been reported in the literature ${ }^{14}$.
Roper and Brenner ${ }^{14}$ have shown that a linear approximation of the Navier-Stokes equations can be useful to accurately determine drag at moderate Reynolds numbers.

Finally, we note the contributions to the force from pressure and viscous terms. Due to separation, the pressure contribution to the thrust force dominates in the thrust causing frozen fin mode. Of the total thrust force of $0.92 \mathrm{mN}$, the pressure contribution is $0.81 \mathrm{mN}$ and the remainder is due to the viscous contribution. In the drag causing slithering mode the viscous contribution is $0.12 \mathrm{mN}$ out of the total drag force of $0.52 \mathrm{mN}$ and the remainder is due to the pressure contribution. Thus, the pressure force dominates thrust while the viscous contribution to drag is relatively larger due to thin boundary layers.
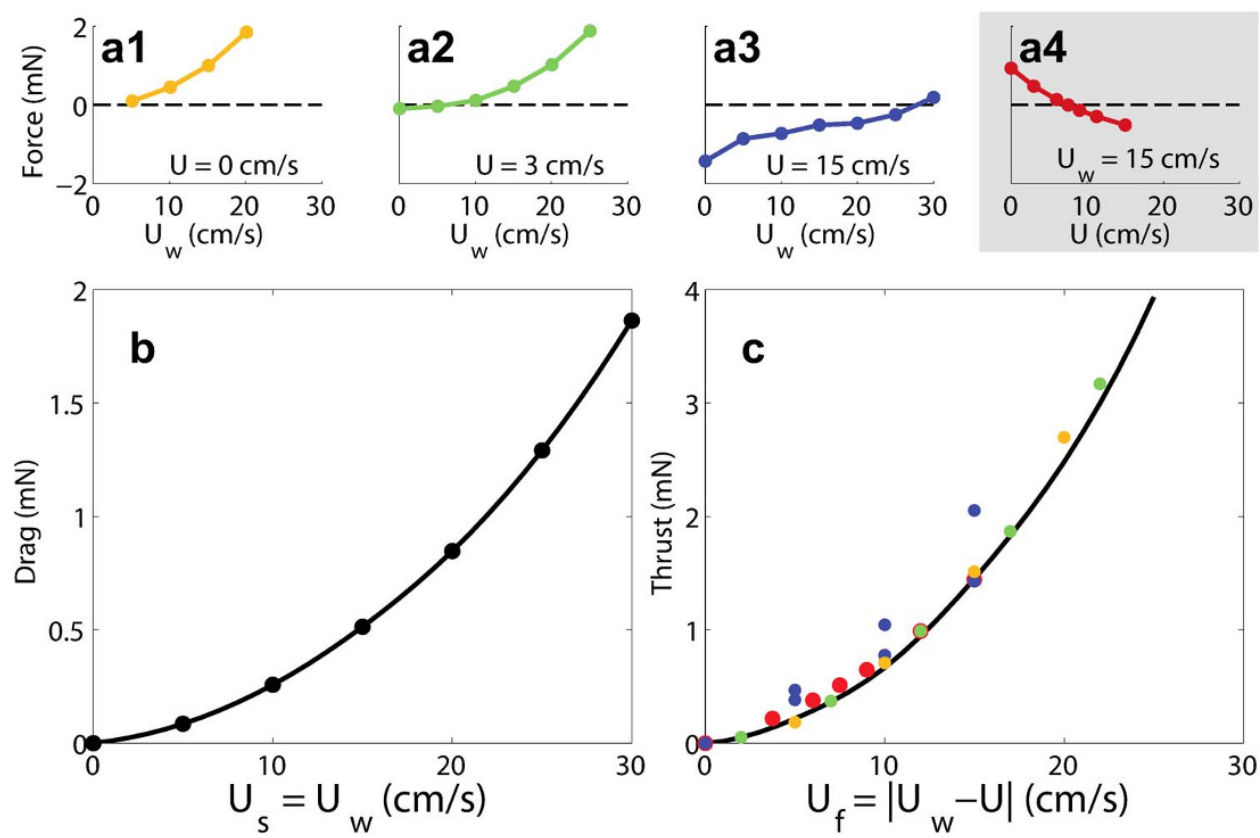

Figure $4 \mid$ (a) The computed total force on the ribbon fin for different parameters. In a1-a3, the body velocity $U$ was held constant as the wave velocity $U_{\mathrm{w}}$ was varied. In a4, the wave velocity $U_{\mathrm{w}}$ was held constant as the body velocity $U$ was varied. The black dotted lines indicating zero total force show where drag and thrust are balanced. (b) Drag, i.e., the force on a ribbon fin during perfect slithering motion. (c) Thrust $T$ computed as a function of $U_{\mathrm{w}}-$ $U$ for each data point in (a) by assuming the kinematic decomposition (Fig. 3 and Eqn. 1). These data are shown by solid dots. Separate frozen fin simulations were conducted as a function of $U_{\mathrm{f}}$, shown by the solid black line. The dots cluster along this line giving evidence for the successful decomposition of drag and thrust. The color of the dots correspond to the data in (a). 


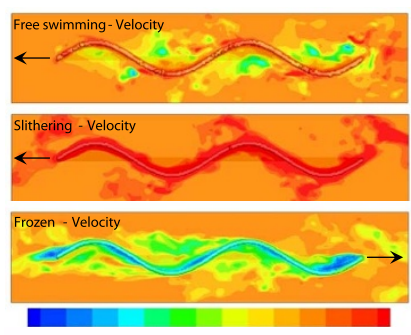

$\begin{array}{llllll}-1.1 & -0.8 & -0.5 & -0.2 & 0.1 & 0.3\end{array}$ Velocity

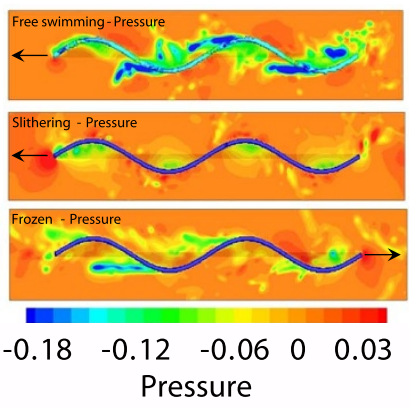

Figure 5 The axial velocity (left) and pressure (right) fields in a crosssectional plane at the bottom edge of the ribbon fin for three cases. Top: Normal case with $U=3 \mathrm{~cm} / \mathrm{s}$ and $U_{\mathrm{w}}=15 \mathrm{~cm} / \mathrm{s}$. Middle: Perfect slithering motion with $U=15 \mathrm{~cm} / \mathrm{s}$ and $U_{\mathrm{w}}=15 \mathrm{~cm} / \mathrm{s}$. Bottom: Frozen fin motion with a backward (i.e. to the right) velocity of $12 \mathrm{~cm} / \mathrm{s}$. The legend for axial velocity (left) show magnitudes that are scaled by $U_{\mathrm{w}}=$ $15 \mathrm{~cm} / \mathrm{s}$. In the contour plot velocity to the left (i.e. forward direction) is positive and to the right (backward) is negative. The legend for pressure (right) show magnitudes scaled by $\rho U_{\mathrm{w}}^{2}$ where $U_{\mathrm{w}}=15 \mathrm{~cm} / \mathrm{s}$.

It has long been hypothesized that the drag of swimming fish is higher due to the thinning of the boundary layers caused by undulatory motion ${ }^{9}$. Fig. 5 shows that the boundary layer flow is a key feature of the drag causing slithering mode. Separated flow plays a role in the thrust mechanism. The separated flow regions are suction zones where the fluid is sucked backward by the undulating fin. This leads to the thrust force. This is consistent with our flow visualization data reported earlier ${ }^{15,16}$.

Generality of the drag-thrust decomposition. The decomposition described above was applied to an idealized ribbon-fin with idealized kinematics at a Reynolds number of $\approx 10,000$. Since this idealization may not be valid for swimming animals, we now examine the applicability of the drag-thrust decomposition to swimming animals with realistic body/fin geometries and measured kinematics. We also examine the validity of the decomposition at moderately high Reynolds numbers by applying the decomposition to a robotic undulatory swimmer ${ }^{17}$.

Application to swimming animals. Our kinematic decomposition of drag and thrust assumed a constant amplitude wave (Fig. 3). This assumption is not strictly valid for swimming animals. For example, in the black ghost knifefish the amplitude of oscillation of the ribbonfin tapers-off toward the two ends ${ }^{13}$ (Fig. 1). Anguilliform and carangiform swimmers have an amplitude that increases with body length ${ }^{18-20}$ (Fig. 1). Additionally, the wave motion may not be strictly sinusoidal. In the non-constant amplitude case, the kinematic split as proposed in this work will not be exact. However, if the amplitude changes are not large then the additional error may not be significant. The approach is expected to work in cases where cross-sections (width of the body in the lateral direction) of the body are nonuniform, provided that the body width does not change sharply along the body. The proposed decomposition is not expected to work for indefinitely high Reynolds numbers, but it does work at moderately high Reynolds numbers which will be demonstrated in the next subsection. Finally, as the height of the ribbon-fin and its amplitude of oscillation is reduced, the drag and thrust producing flow fields may not remain as separate as the case shown in Fig. 5. Thus, as the undulatory propulsor becomes slender (length of a fin ray shown in Fig. 2c approaches zero), the decomposition of drag and thrust may not be as clear. Given these issues, we examine the applicability of the decomposition with simulations of the eel Anguilla rostrata, the larval zebrafish Danio rerio, the black ghost knifefish Apteronotus albifrons, and the mackerel Scomber scombrus. These examples show where the decomposition is valid, and where it becomes invalid.

The drag, thrust, and undecomposed forces on a black ghost knifefish (gymnotiform), an eel (anguilliform), a larval zebrafish (subcarangiform), and a mackerel (carangiform) are tabulated in Table 1. Table 1 shows that the error in decomposing forces on free swimming knifefish, eel, and zebrafish is small compared to that in decomposing the force on a mackerel. During steady free swimming, the average force in the swimming direction is zero. For a steady free swimming organism, the drag and thrust components must be equal. For the black ghost knifefish and the larval zebrafish, it is seen that the drag and thrust forces are equal to each other within $5 \%$. Note that the knifefish displays a small variation in amplitude whereas the zebrafish has a subcarangiform amplitude variation. For the eel, which has an anguilliform amplitude variation, the drag and thrust forces are equal with an error of $17 \%$ (Table 1). Thus, we see that our decomposition works well for gymnotiform (and by similarity for balistiform and rajiform), anguilliform, and subcarangiform swimmers, none of which display rapid variations in amplitude. But, the decomposition does not work well for carangiform swimmers because the rate of change of amplitude in these swimmers is very high (Table 1). An assessment of why the decomposition works well for modest variations in body or fin amplitude along the body length, but not for large variations in amplitude, is presented in the Discussion Section.

Application to a robotic knifefish. Here we examine how well the decomposition works at moderately high Reynolds numbers. We consider parameters for a robotic knifefish ${ }^{17}$, approximately three times longer than the adult live knifefish. At typical kinematic parameters, such as two undulations along the fin undulating at $2.5 \mathrm{~Hz}$,

Table 1 | Drag-thrust decomposition of free swimming black ghost knifefish fin, eel, larval zebrafish. Also shown is the decomposition of a hypothetical fin with mackerel kinematics. The amplitude shown for the knifefish is at distance of $0.75 \mathrm{~cm}$ from base of the fin. The range for $x$ in $A(x)$ is $[0,1]$. Undecomposed forces equal to zero indicate free swimming cases

\begin{tabular}{|c|c|c|c|c|}
\hline & Knifefish & Eel & Zebrafish (larval) & Mackerel \\
\hline Amplitude (cm) & $\begin{array}{ccc}-0.3 & & \\
-0.5 & 4.5 & 9\end{array}$ & -0.2 & $\begin{array}{ll}-0.1 & 0.2 \\
x\end{array}$ & 0.1 \\
\hline $\begin{array}{l}\text { Amplitude function } A(x) \\
\text { Thrust force } T(m N) \\
\text { Drag force } D(m N) \\
\text { Undecomposed force } F(m N) \\
\text { Net force } D-T(m N)\end{array}$ & $\begin{array}{c}\text { Experimental }^{13} \\
1.43 \\
1.48 \\
0 \\
0.05\end{array}$ & $\begin{array}{l}0.15 e^{(x-1) 19} \\
6.8 \times 10^{-4} \\
8.2 \times 10^{-4} \\
0 \\
1.4 \times 10^{-4}\end{array}$ & $\begin{array}{c}\text { Experimental } \\
4.3 \times 10^{-3} \\
4.1 \times 10^{-3} \\
0 \\
-0.2 \times 10^{-3}\end{array}$ & $\begin{aligned} 0.02- & 0.08 x+0.16 x^{218,20} \\
& 11.6 \times 10^{-3} \\
& 46.8 \times 10^{-3} \\
& 21.4 \times 10^{-3} \\
& 35.2 \times 10^{-3}\end{aligned}$ \\
\hline
\end{tabular}


the Reynolds number based on fin length is around 130,000. Decomposition of drag and thrust forces on ribbon-fins at the scale of the robot was tested using simulations and experiments with the robot. The fin has the same dimensions and kinematic parameters in the simulation and the experiment. However, the surface of the experimental fin departs from the simulated fin in a manner that appears to affect our results, as will be described later.

Simulations of the decomposition were carried out for a fin the same size as that on the robot, but without a body. In Section S2 and Fig. S2 of SI we show that the forces on the body and the fin are decoupled, and the presence of the body has no influence on the fin forces. Hence, the decomposition can be carried out with or without the body. We choose to carry out the decomposition without the body to reduce the computational cost. We consider a case where $U=0 \mathrm{~cm} / \mathrm{s}$ and $U_{\mathrm{w}}=40.75 \mathrm{~cm} / \mathrm{s}$. The net force generated by the fin was found to be $384.7 \mathrm{mN}$. In the drag causing slithering mode, the fin translated forward at $U=U_{\mathrm{w}}=40.75 \mathrm{~cm} / \mathrm{s}$. In the thrust-causing frozen mode the fin translated backward with a velocity of $U_{\mathrm{w}}-U=$ $40.75 \mathrm{~cm} / \mathrm{s}$ (slithering and frozen velocity are same because $U=$ $0 \mathrm{~cm} / \mathrm{s}$ in the undecomposed mode). Thrust and drag forces were found to be $427.1 \mathrm{mN}$ and $33.54 \mathrm{mN}$, respectively. The difference between thrust and drag force $(393.46 \mathrm{mN})$, matches well with the force of the undecomposed mode $(384.7 \mathrm{mN})$. Based on simulations, we infer that the drag-thrust decomposition is valid at length scales of robotic knifefish's ribbon-fin.

As noted before, the parameters used in the experiments were same as those used in simulations, above. Forces in the undecomposed, slithering (drag), and frozen (thrust) modes were measured to be $226.8 \mathrm{mN}, 229.5 \mathrm{mN}$, and $283.3 \mathrm{mN}$ respectively. The difference between drag and thrust force is not equal to the undecomposed force. The undecomposed force and thrust force are of the same order of magnitude just as it is in simulations. This raises two important questions: i) what is the source of the larger than expected slithering (drag) mode force? ii) given this disagreement, is there a resolution? These questions will be addressed in the Discussion Section.

Utility of the drag-thrust decomposition. Optimal height of a knifefish ribbon fin. What is the utility of separating drag and thrust in the manner we have proposed? Next we show that this decomposition provides a powerful predictive tool. To that end, we consider a specific example problem: given the body of a knifefish, which is held nearly rigid, what should be the height of its ribbon fin? We also show how drag-thrust decomposition leads to models that can predict the swimming velocity of an organism.

To find the preferred fin height, we hypothesized that the observed height of the ribbon fin is such that the mechanical energy spent per unit distance traveled, referred to as the mechanical cost of transport (COT), is minimized. The COT was computed numerically for different fin heights as discussed below. We considered steady swimming in which a fish moves with a constant mean velocity. For simplicity, we considered a plate-fin configuration like that used by Lighthill and Blake ${ }^{11}$ to study gymnotiform and balistiform swimming. A plate of height $s=2 \mathrm{~cm}$ and length $L=10 \mathrm{~cm}$ was attached to a ribbon fin of the same length (Fig. 6). These dimensions were selected based on typical fin and body heights in adult knifefish ${ }^{12,13,21}$. The following kinematic parameters were chosen: $\theta_{\max }=30^{\circ}, f=$ $3 \mathrm{~Hz}, \lambda=5 \mathrm{~cm}$. The fin height was varied from $0.5 \mathrm{~cm}$ to $2.5 \mathrm{~cm}$. For each fin height we solved the problem of self-propulsion by using a previously developed efficient algorithm ${ }^{22}$. In these computations the traveling wave motion of the ribbon fin attached to the plate was specified. For each case we computed the mean power $P$ spent by the fin against the fluid over one period of the steady swimming cycle. The time-averaged swimming velocity $U_{s}$ was estimated during steady swimming. The cost of transport was computed as COT $=$ $P / U_{\text {s. }}$.

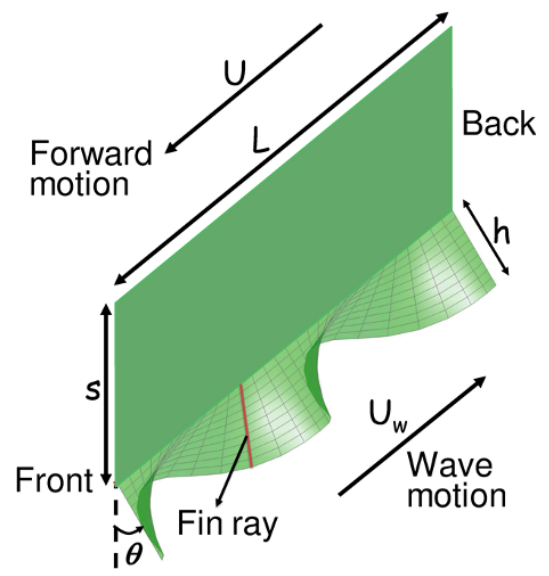

Figure $6 \mid$ Geometric parameters and the configuration of the plate-fin assembly.

Fig. 7 shows plots of the swimming velocity $U_{s}$, the mean power $P$, and COT as a function of the ribbon fin height $h$. The power spent on the fluid follows a power law trend (Fig. 7a). The swimming velocity $U_{\mathrm{s}}$ first increases rapidly with respect to $h$ and then changes slowly at higher values of $h$ (Fig. 7b). This trend is a direct result of different scalings of drag and thrust forces with respect to $h$ (Section S2 of SI). The COT is low and nearly constant at smaller $h$ after which it grows rapidly (Fig. 7c). The basis of this increase in COT is that at larger $h$, the power increases with increasing $h$ but the corresponding increase in $U_{s}$ is small. Hence there is a rapid growth of COT at larger $h$. Fig. 7c shows that the ribbon fin heights that give lower values of COT, for a plate height of $2 \mathrm{~cm}$, are in the range of $0.5-1.1 \mathrm{~cm}$. In this range the COT does not change significantly but the swimming velocity is highest at $h=1.1 \mathrm{~cm}$. In short, different scalings of drag and thrust with respect to $h$ lead to a specific trend of $U_{s}$ vs. $h$, which in turn determines the trend of COT vs. $h$. The COT trend eventually provides the prediction for the fin height $h$ that will minimize the metabolic cost of movement, and as we will see in the next section, this predicted height agrees well with observed fin heights.

Sensitivity of the optimal fin height to fish body size: The predicted fin height $(\sim 1 \mathrm{~cm})$ is consistent with the mean fin height of $0.97 \mathrm{~cm}$ that we measured for 13 species in 8 genera in the family Apteronotidae of weakly electric South American knifefishes (Table 2, at $50 \%$ body length). The standard deviation of the fin height from the measured mean value (blue vertical bar in Fig. 7c) is within the range of fin heights $(0.5-1.1 \mathrm{~cm})$ for which COT is predicted to be low. Although the body height of the fishes we considered did vary, we found from a sensitivity analysis (see Section S3 of SI) that the influence of the plate (or body) height on the COT trend is not significant. We show this result in Fig. 7c, where the red shaded region shows the variation in COT due to a change in plate height corresponding to the standard deviation in the body height of the 13 species we measured.

Prediction of swimming velocity. Finally, to show that the proposed drag-thrust decomposition can be used to predict swimming velocities, a force balance equation similar to Eqn. 1 was written for the steadily swimming fin-plate assembly (Eqn. S6 of SI). We used that equation to derive an analytic solution for the fin height as a function of swimming velocity (Eqn. S8 of SI), which can be rearranged to give swimming velocity as a function of fin height. To test the analytic prediction of swimming velocity we performed numerical simulations to compute the swimming velocity. Excellent agreement between the analytic and numerical solutions of swimming velocity is shown in Fig. $7 \mathrm{~b}$.

\section{Discussion}

Are there other ways to obtain drag-thrust decomposition? There can be many kinematically consistent decompositions which satisfy 

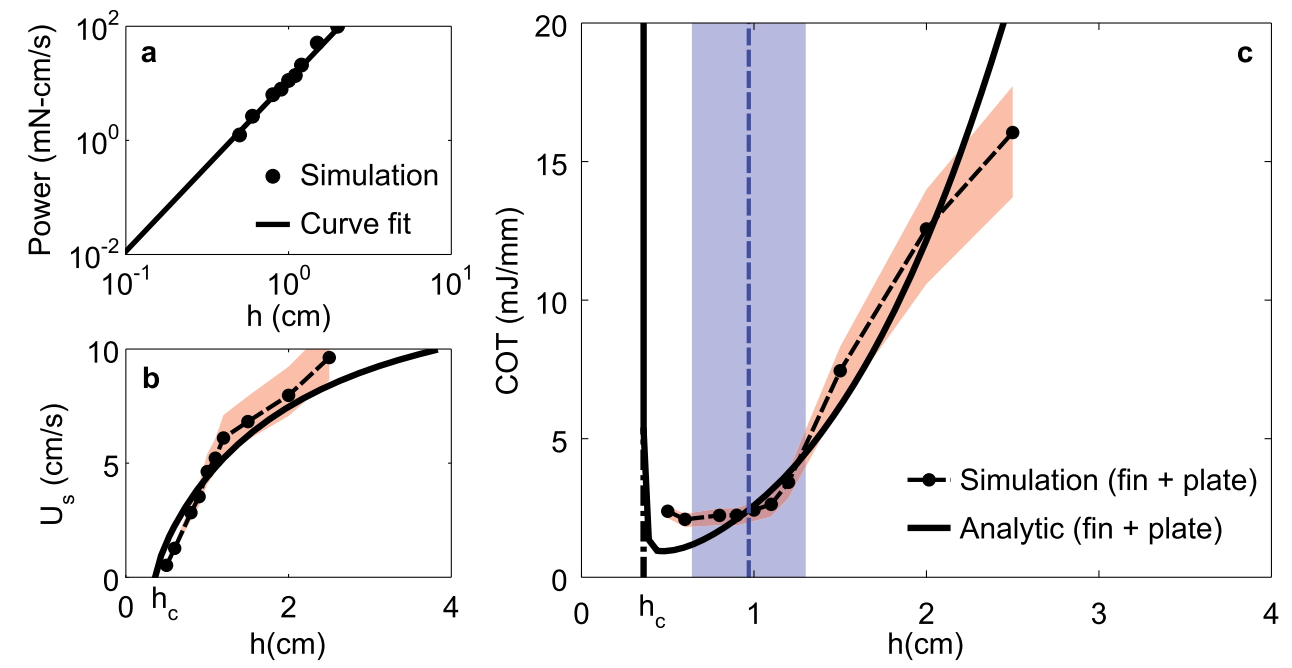

Figure $7 \mid$ (a) Mechanical power expended in the fluid, obtained from fully resolved simulations of self-propulsion, as a function of the fin height. (b) Swimming velocity as a function of the fin height $h$ obtained from fully resolved simulation of self-propulsion as well as from a reduced order model (solid line). (c) The mechanical cost of transport as a function of the fin height $h$ obtained from fully resolved simulation of self-propulsion as well as from a reduced order model. The red shaded region represents the variation of swimming velocity in (b) and cost of transport in (c) due to a perturbation to the plate height. The perturbation to the height is equal to $\pm 0.85 \mathrm{~cm}$, which corresponds to the standard deviation of the measured body heights in an assortment of knifefish at the half way point along the fin (see Table 2). The dashed blue vertical line in (c) corresponds to the mean fin height measured, and the blue shaded region represents the standard deviation in fin height. Note: The closed circle and the solid line in (b) and (c) have the same meaning.

kinematic conditions D1 (body movements creating drag summed to body movements creating thrust result in the original undecomposed movement) and D2 (body movements creating drag and thrust are without discontinuities and physically realizable). However, the kinematic decomposition depicted in Fig. 3 is the only one we have found satisfying D1 and D2 as well as providing force decomposition (D3, the sum of the decomposed drag and thrust forces is equal to force in the originally observed swimming motion of the animal). The primary reason that force decomposition becomes possible is due to boundary layer flow in the drag mechanism and separated flow in the thrust mechanism (Fig. 5). A boundary layer flow is observed in the drag mechanism because in that case the velocity on the fin surface is tangential to the surface itself (Fig. 5). This type of internal boundary condition arises because the forward translational velocity of the fin in the slithering mode is equal and opposite to the wave velocity of the undulating fin. Any translational velocity in the drag mode that is other than $U_{\mathrm{w}}$ will result in a velocity at each point on the fin that is no longer tangential to the surface. This gives rise to a flow field that is not purely due to the boundary layer and it couples with the separated flow field due to the thrust mechanism. Thus, even if other decompositions are kinematically correct, the force decomposition will not work well. The kinematic decomposition that we have is the one that leads to the least coupling between the drag and thrust modes for the kinematics considered here.

Table 2 | Body length (BL), body length with filament (BLF), fin length (FL), body height (S), fin height (H), ratio of body height to fin height (R) of 13 species across 8 genera of South American weakly electric fishes in the Apteronotidae, in order of body height at $50 \%$ along fin length. Specimen identification numbers (Harvard Museum of Comparative Zoology) are represented by Sp. ID. Body height, fin height, and their ratio (R) were measured at 25,50, and 75 percent along the fin length, and the same are tabulated. Note: All lengths are in centimeters, asterisk indicates that the quantity in question was estimated, not measured

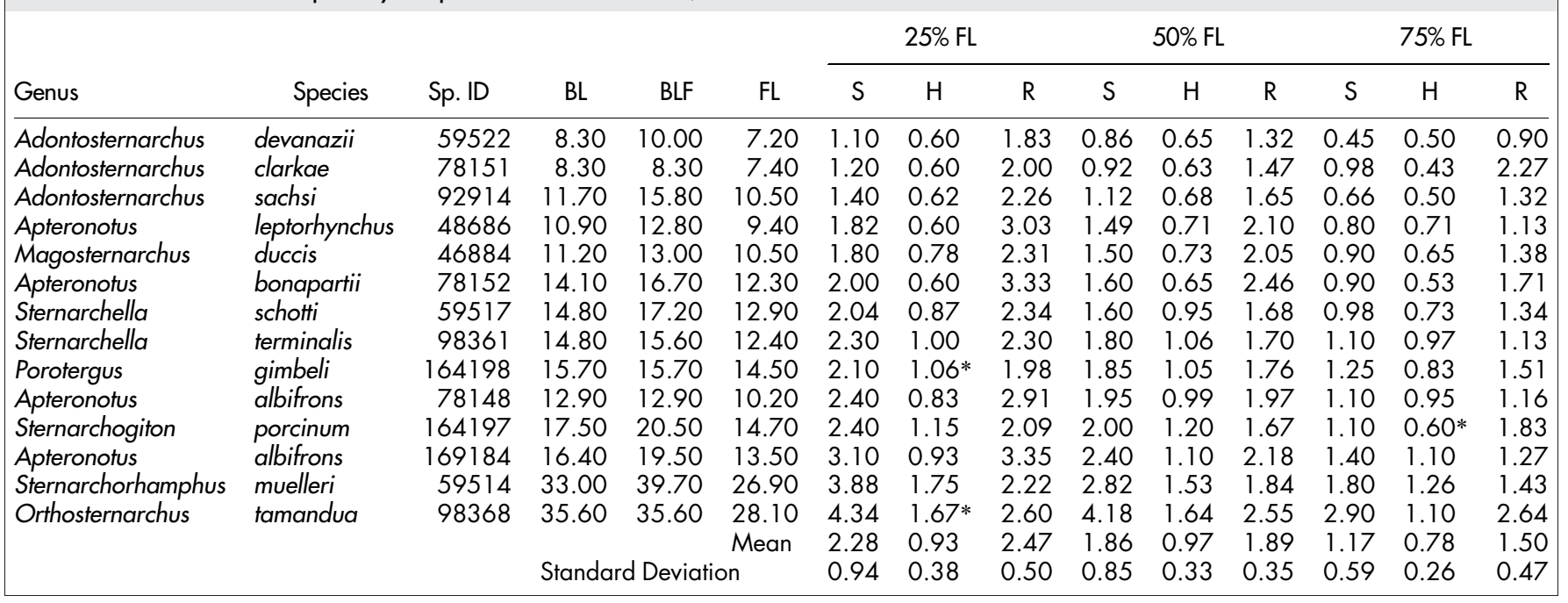


As an example, consider a different kinematic decomposition where the backward traveling wave is defined as the thrust producing mechanism whereas the fin translating forward with velocity $U$ is defined as the drag producing mechanism. We used our data to check if this kinematic decomposition also leads to force decomposition. If valid then it should be possible to split the total force as

$$
F\left[U, U_{\mathrm{w}}\right]=T_{\mathrm{s}}\left[U_{\mathrm{w}}\right]-D_{\mathrm{f}}[U],
$$

where $T_{\mathrm{s}}\left[U_{\mathrm{w}}\right]$ is the forward force on a stationary fin with traveling waves moving backward with wave velocity $U_{\mathrm{w}}$, and $D_{\mathrm{f}}[U]$ is the force on a fin with a fixed shape, i.e. the frozen fin, that is translating forward with velocity $U$. Using the data for $F$ in Fig. 4a1-a4 and the data for $D_{\mathrm{f}}[U]$, available from our frozen fin simulations, we computed $T_{\mathrm{s}}\left[U_{\mathrm{w}}\right]$. These values are plotted in Fig. 8 and compared to the force on a stationary ribbon-fin from our prior work ${ }^{15}$. If this decomposition is valid, all data should fall on a single curve in Fig. 8. That is not the case. It can be shown that the reason this decomposition does not work is because the corresponding flow fields are not decoupled.

Given that drag and thrust appear intermingled in swimming organisms, especially in the undulatory mode, it has been hypothesized that there must be some spatial or temporal separation between thrust and drag production that allows the total force to be zero on average over a swimming cycle $^{19}$. A model to estimate thrust based on temporal oscillations of the swimming velocity has been proposed ${ }^{23}$. Our data suggests that a decomposition of the total force into drag and thrust is possible without relying on spatio-temporal splitting.

Comments on different measures of drag reported in literature. Appropriate measures of drag on a swimming organism have been debated in literature for many decades ${ }^{24,25}$. Here, we discuss how drag from our decomposition is differs from definitions of drag in the literature. One measure that has been used is the tow-drag, i.e., the drag on a non-swimming organism if it is pulled in the fluid at its swimming velocity. The organism is usually not deformed in these experiments or theoretical estimates. This drag measure is not expected to be correct because the shape of the animal for the towdrag estimate does not match with shape of the fish during propulsive movement ${ }^{24}$. The second measure is the drag obtained by pulling a deformed non-undulating body through the fluid at its swimming velocity, i.e., the drag on the frozen shape configuration. As noted above and in Fig. 8, this does not result in successful decoupling of thrust from drag. Our results suggest that an appropriate drag

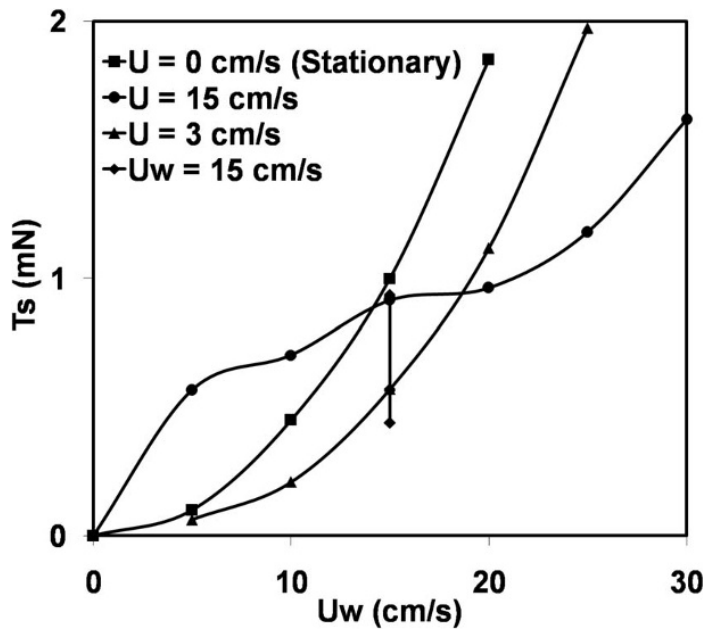

Figure 8 The thrust force $T_{\mathrm{s}}$ computed as a function of $U_{\mathrm{w}}$ for each data point in Fig. 3 by assuming a decomposition according to Eqn. 2. In this case the data do not cluster along a single curve. The legend identifies different simulation sets. For example, the set with $U_{\mathrm{w}}=15 \mathrm{~cm} / \mathrm{s}$ was the one where $U_{\mathrm{w}}$ was fixed and the value of $U$ was changed. measure for undulatory propulsion is the one corresponding to the perfect slithering motion at the wave velocity (Fig. 5).

These measures are best illustrated by considering a hypothetical swimming ribbon-fin with no body attached to it. According to the drag-thrust decomposition and the data in Figs. $4 \mathrm{~b}$ and $4 \mathrm{c}$, a ribbon-fin with $U_{\mathrm{w}} \approx 22 \mathrm{~cm} / \mathrm{s}$ will swim with a velocity $U \approx$ $9.5 \mathrm{~cm} / \mathrm{s}$. The first drag measure - the tow-drag - for this case corresponds to the drag on a flat plate towed at $9.5 \mathrm{~cm} / \mathrm{s}$. This is estimated to be $0.12 \mathrm{mN}$ based on boundary layer theory. The second drag measure corresponding to a frozen fin, moving at $9.5 \mathrm{~cm} / \mathrm{s}$, is $0.6 \mathrm{mN}$. Finally, the third drag measure proposed by us corresponding to the perfect slithering motion with $U_{\mathrm{w}} \approx 22 \mathrm{~cm} / \mathrm{s}$ is $1 \mathrm{mN}$. Thus, our drag measure is higher than the other two estimates for the scenario considered here. The result is consistent with reports in literature that the tow-drag is often found to be lower than that required to achieve a balance of drag and thrust forces during swimming ${ }^{24,25}$. In general, however, the relative magnitudes of the three drag measures may not be in the same order as in the example discussed above. It will depend on various parameters including the geometric configuration.

It has been noted in the past that body undulations lead to a reduction in drag on a swimming body ${ }^{26}$. That conclusion was based on computing the total force on an infinite two-dimensional wavy surface for a given imposed velocity $U$ and then noting that as $U_{\mathrm{w}}$ is increased the total force changes from being backward (drag-like) to being forward (thrust-like). In this sense the presence of undulations reduces the drag-like behavior. This is consistent with our results.

Limits of the drag-thrust decomposition. Results of the decomposition of forces on swimming animals showed that the decomposition is valid for swimming animals with anguilliform, gymnotiform (by similarity balistiform and rajiform), and subcarangiform kinematics. But, the decomposition is not valid for swimming animals with carangiform kinematics. Here, we present a theoretical assessment of when the proposed decomposition will be correct and when it will fail. For the purpose of analysis, consider kinematics imposed on a rectangular surface ("fin" hereafter) of infinitesimal thickness. At any instant a given point on the fin undergoes lateral displacement given by

$$
y=A(x) \sin \left[2 \pi\left(\frac{x}{\lambda}+f t\right)\right],
$$

where $A(x)$ is the amplitude which is a function of axial direction and it determines the mode of swimming. The amplitude for different modes of swimming is shown in Table 1. The deviation from exact decomposition is a function of the rate of increase of amplitude with body length. The deviation will be more if the rate of amplitude change is high and vice-versa. This will be demonstrated below.

The slithering mode is affected by a deviation from exact decomposition. The slithering mode has the property that velocity at each point on the fin is tangential to surface of the fin. The velocity in the slithering mode, at a point, is the resultant of the forward translational velocity $U_{\mathrm{w}}$ and the lateral velocity $V_{\mathrm{w}}$. The angle, $\alpha$ (Fig. 3), of the velocity at a point is given by

$$
\tan \alpha=\frac{2 \pi A(x) \cos \left[2 \pi\left(\frac{x}{\lambda}+f t\right)\right]}{\lambda} .
$$

The direction of the resultant velocity at every point on the fin surface must be equal to slope of the corresponding point if the velocity has to be tangential to the fin surface. The slope of a point on the fin undergoing traveling wave motion is given by

$$
\frac{d y}{d x}=\frac{2 \pi A(x) \cos \left[2 \pi\left(\frac{x}{\lambda}+f t\right)\right]}{\lambda}+\frac{d A(x)}{d x} \sin \left[2 \pi\left(\frac{x}{\lambda}+f t\right)\right] .
$$

Note that if $A(x)$ is constant then Eqn. 4 and 5 are identical resulting in a perfect slithering motion where the resultant velocity of a point is 
tangential to the fin surface. Substituting Eqn. 4 in 5 we get the following equation

$$
\frac{d y}{d x}-\tan \alpha=\frac{d A(x)}{d x} \sin \left[2 \pi\left(\frac{x}{\lambda}+f t\right)\right] .
$$

The above equation is a measure of deviation from exact decomposition. At a given instant of time, the deviation from tangential velocity is directly proportional to $\frac{d A(x)}{d x}$. The figures in the first row of Table 1 show that the rate of amplitude change in anguilliform swimmers, subcarangiform swimmers and knifefish is slower than that in carangiform swimmers. In anguilliform swimmers the amplitude gradually increases from head to tail, i.e, the rate of change of amplitude is smaller. Thus, the deviation from perfect slithering motion is small. The same is true for the subcarangiform swimmer as well. In case of the knifefish amplitude, the amplitude first increases, reaches a peak, and then decreases. But the rate at which amplitude increases or decreases is very small, hence the deviation from perfect slithering motion is small. Thus, the decomposition of eel, larval zebrafish and knifefish worked well. However, in carangiform swimmers, the amplitude remains relatively small and constant for at least half the body length after which it increases rapidly. The rate of change of amplitude is very high towards the caudal portion of the body thus resulting in a large deviation from perfect slithering motion. Consequently, it is not surprising that the decomposition did not work for mackerel.

Why the drag-thrust decomposition failed for the robotic knifefish. Using numerical simulations it was shown that the decomposition was valid for the robotic knifefish. But, experimental data did not agree with the simulations. The main difference between the experiments and the simulation was in the force of the slithering mode. The slithering drag force from experiment was higher than that from simulation; this implies that the drag force is higher than what it should be for the decomposition to be valid. We hypothesize that the larger than expected slithering drag force in the experiment is due to the imperfections on the robot's fin surface. The robotic fin is made up of discrete rays (32 rays $1 \mathrm{~cm}$ apart) that are connected by Lycra fabric; see Fig. 9 in which one of the fin rays is highlighted in white. It is not possible to produce a smooth sinusoidal wave on the fin unless the fin is made up of very large number of rays. Owing to the limited number of rays, the sinusoidal wave generated by the robotic fin is not smooth (see Fig. 10). With kinks on its surface the robotic fin cannot maintain a thin boundary layer in the slithering mode like that in simulations. The kinks will introduce disturbances into the boundary layer (see Fig. 11). Unlike its real counterpart, the robotic fin modeled in the simulation is composed of very fine grid points (whose resolution is of the order of the fluid grid resolution).

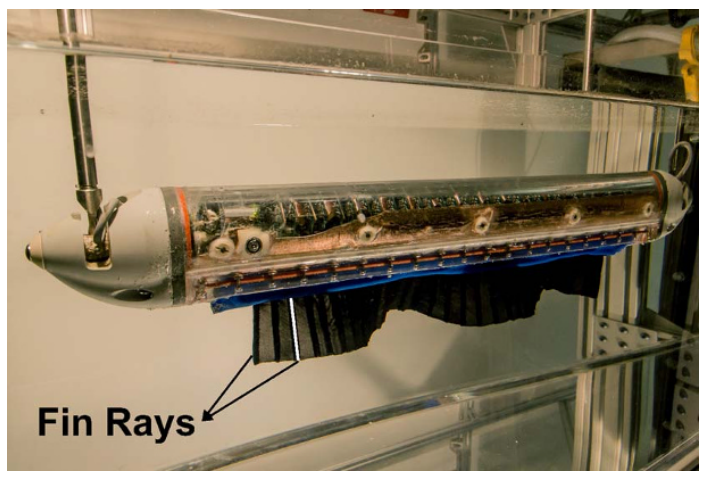

Figure 9 | The robotic knifefish used in the drag-thrust decomposition experiments. The robotic ribbon-fin is composed of 32 fin rays and a Lycra fabric connecting the rays. One of the fin rays is coloured white to highlight it.

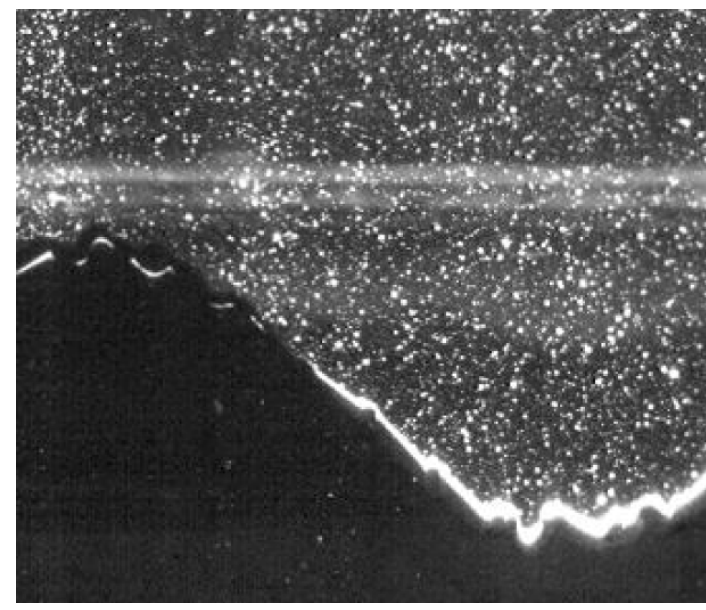

Figure 10 The surface of the robotic ribbon fin during sinusoidal undulations. The material between adjacent rays folds and results in kinks on the fin surface.

Any curved surface can be imposed (sinusoidal or otherwise) on the modeled fin surface without causing any kinks. Hence, it leads to a thin undisturbed boundary layer in the slithering mode (see Fig. 11). The boundary layer on the robotic fin is very thick when compared to that from simulation or that from flow past a flat plate at the same Reynolds number (see Fig. 11). The large thickness of the boundary layer may also indicate separated flow, which could lead to the large drag force measured in the experiment.

An alternate estimate of the drag force on the robotic ribbon fin. Given that the slithering mode force in the experiment does not satisfy the drag-thrust decomposition due to disturbances caused by surface kinks, we propose that the next best choice may be to use a drag estimate that is similar to the tow-drag. This is because the surface imperfections on an undeformed (or straight) robotic ribbon-fin would be much less and consequently the flow would be less perturbed or separated. There is subtle, yet important, difference between our estimate of drag and the conventional measure of tow-drag. In the conventional measure of tow-drag, the swimmer is towed at its swimming speed in stationary water. In contrast, we measure the drag by towing a straight fin, in stationary water, at the velocity of the slithering mode (which is equal to wave velocity, $U_{\mathrm{w}}=40.75 \mathrm{~cm} / \mathrm{s}$ ) instead of swimming

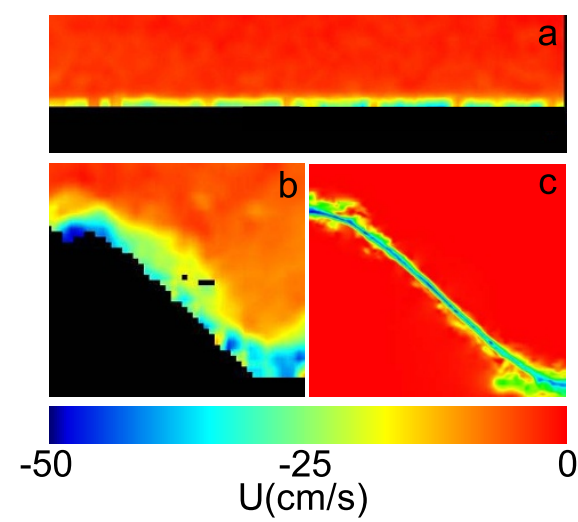

Figure 11 A comparison between the boundary layer due to, a) flow past a flat plate, b) slithering motion of a robotic ribbon fin, c) and slithering motion of a modeled robotic fin in a simulation. The boundary layer is shown on a horizontal plane $1 \mathrm{~cm}$ above the bottom edge of the fin toward the robot body. The color bar represents the axial velocity. 
speed. The force was measured to be $81.9 \mathrm{mN}$. This value is closer to the expected value of $56.6 \mathrm{mN}$ obtained from the drag thrust decomposition estimate.

Why the drag-thrust decomposition works for real knifefishes. Simulations have shown that the decomposition is indeed valid at moderately high Reynolds number. Experiments on the robotic knifefish, however, suggest possible limitations of the decomposition. The decomposition is sensitive to any deviation from perfect slithering mode, be it due to high levels of amplitude change or high roughness on the fin surface. A knifefish's ribbonfin is composed of 120-320 rays spanning fin lengths of $10-30 \mathrm{~cm}$, giving typical densities of about one ray per millimeter (Table 4 and Fig. 41 of ref. 27). Many of these fin rays branch into two rami half the way from their base to their distal ends, which would effectively double the ray density along the very portion of the fin surface that could become uneven due to spreading between the rays when they are oscillated ${ }^{27}$. With such fine ray spacing, a knifefish can produce curved surfaces on its fin without kinks. In contrast, on the robot the ray density is one ray per $10 \mathrm{~mm}$, resulting in a much less smooth fin surface. We therefore expect that drag-thrust decomposition would be applicable to a robotic ribbon-fin if it can be designed to have higher ray density so that the fin surface is smoother.

\section{Methods}

Experimental setup. Robotic undulating fin. The robotic model used for the experimental work was the 'Ghostbot,' a biomimetic knifefish robot which undulates an elongated fin to generate thrust (see Fig. 9). The robot consists of a rigid cylindrical body that houses the motors and electronics to drive the individual rays of the fin. There are 32 rays to actuate a rectangular Lycra fin measuring $32.6 \mathrm{~cm}$ by $5 \mathrm{~cm}$. More details of the robot are found $\mathrm{in}^{17}$, with the only difference being the depth of the fin, which was $3.37 \mathrm{~cm}$ in the previous work rather than $5 \mathrm{~cm}$ for the fin used in the experiments discussed here.

Measuring hydrodynamic forces. The robot was suspended horizontally into a variable speed flow tank from an air-bearing platform allowing near frictionless motion in the longitudinal axis. We fixed the robot in the lateral and vertical directions. We placed a single axis force transducer (LSB200, Futek, Irvine, CA, USA) in the longitudinal axis between the air-bearing platform and mechanical ground, allowing us to measure the forces generated by the robot or acting on the robot along that axis. Voltages from the force transducer were recorded at $1000 \mathrm{~Hz}$. For each trial, we allowed ample time for the hydrodynamics to reach steady state (30-60 seconds), then averaged the last 10 seconds of data, which was converted to force units based on the calibration, which had a maximum nonlinear error of $0.034 \%$. The flow speed of the water tunnel was measured and calibrated using particle image velocimetry (PIV). More details on PIV are provided in the following section.

Particle image velocimetry (PIV). We analyzed horizontal PIV planes to measure the boundary layer thickness caused by the fin. The PIV setup used is the same as the one described in ${ }^{16}$. In short, a 2 W laser beam (Verdi G2, Coherent Inc., Santa Clara, CA, USA) is scanned at $500 \mathrm{~Hz}$ to create a planar laser light sheet. A high-speed camera (FastCam 1024P PCI, Photron, San Diego, CA, USA) imaged reflective particles suspended in the fluid ( 44 micron silver coated glass spheres, Potter Industries, Valley Forge, PA, USA) at 500 frames per second, matching the scanning rate of the laser. High-speed video was analyzed using a commercial software package (DaVis, LaVision GMBH., Göttingen, Germany). Successive frames of the video were crosscorrelated to calculate the velocity vector field of the fluid. Cross-correlation consisted of two passes with decreasing interrogation windows, first with an interrogation window of 32 by 32 pixels with 50\% overlap and second with a window of 16 by 16 pixels with $50 \%$ overlap.
Numerical problem formulation. For the numerical simulations, the ribbon fin is modeled as a thin membrane as shown in Fig. 2. The angular position $\theta(x, t)$ of any point on the fin (described above in under Results) is modeled as

$$
\theta(x, t)=\theta_{\max } \sin 2 \pi\left(\frac{x}{\lambda}-f t\right) .
$$

This corresponds to a sinusoidal traveling wave along the fin of length $L$ and height $h$. Kinematic parameters are frequency $f, \theta_{\max }$, and $\lambda$. The speed at which the wave form travels along the fin is called the wave velocity, $U_{\mathrm{w}}=f \lambda$. In the optimal ribbon fin height analysis (presented in Results section), the knifefish is modeled as a plate-fin assembly where a rigid plate is attached to the ribbon-fin in place of the fish's rigid body. In these simulations, the rigid plate is modeled as a rigid surface. The properties of water are used for the fluid. Two types of simulations are performed in this work. In one type, the translational velocity $U$ of the fin and/or plate is specified along with the deformation kinematics of the fin (Eqn. 7). These simulations are carried out in the frame of reference of the fin/plate. Hence the translational velocity $U$ appears as an imposed free stream velocity. Rotation of the fin/plate is prohibited.

In the second type of simulation, only the deformation kinematics of the fin are prescribed, which result in a self-propelling fin-plate assembly. Complete details of the computational method and validation are given in refs. 15, 22. In this method, the viscous Navier-Stokes equations along with the incompressibility constraint are solved in the entire domain. The effect of the immersed fin/plate is resolved by a new constraint based formulation described $\mathrm{in}^{22}$. A finite difference method that is $6^{\text {th }}$ order in space and $4^{\text {th }}$ order in time is used. The grid size was chosen after performing a grid-sensitivity study. The Courant-Friedrichs-Lewy (CFL) number is 0.25 for all simulations. Periodic boundary conditions are used in all directions. The computational domain was made large enough to minimize the impact of periodicity. Mean forces and power of the fin were calculated as the time average over at least one period of oscillation, after a quasi-steady state is reached.

Parameters chosen to match those of an adult black ghost knifefish ${ }^{12}$ are: fin length $L=10 \mathrm{~cm}$, fin height $h=1 \mathrm{~cm}, f=3 \mathrm{~Hz}, \theta_{\max }=30^{\circ}$, and $\lambda=5 \mathrm{~cm}$. The density and viscosity of water are taken as $\rho=1,000 \mathrm{~kg} / \mathrm{m}^{3}$ and $\mu=8.9 \times 10^{-4} \mathrm{~kg} / \mathrm{m} \cdot \mathrm{s}$ unless otherwise specified.

Numerical simulations of swimming animals. Real three dimensional geometries of the bodies or fins were simulated in all cases except the mackerel where a sheet-like fin was simulated with mackerel-like kinematics. The simulation parameters and the kinematics are given in Table 3. The fin profile and the kinematic data of the knifefish were experimentally obtained by us ${ }^{13}$. Only the ribbon-fin of the knifefish was considered for the decomposition. The body of the knifefish was not considered for the same reason the body was not considered in the robot simulation. Experimentally extracted kinematic data and body profile of the larval zebrafish were provided by Melina Hale of The University of Chicago. The body profile of the eel was taken from Kern and Koumoutsakos ${ }^{28}$ analysis of anguilliform swimming. The kinematics of both the mackerel and eel were described by Eqn. 3, where the amplitude of kinematic undulations are based on experiments. Eel kinematics were based on experiments by Tytel and Lauder ${ }^{19}$, and mackerel kinematics were based on experiments by Videler and $\mathrm{Hess}^{18}$

Sign convention. Refer to Fig. 3 and Fig. 6 . The forward direction is to the left and the backward direction is to the right.

Translational velocities $U$ and $U_{s}$, of the swimming body or the fin, are positive if directed to the left (forward). The wave velocity $U_{\mathrm{w}}$ is positive if directed to the right (backward). The frozen fin velocity $U_{\mathrm{f}}$ is positive if directed to the right (backward). In Fig. 3, $U_{\mathrm{s}}=U_{\mathrm{w}}$ for the slithering mode implies that the translational velocity $U_{\mathrm{s}}$ is positive and directed to the left (forward) when the wave velocity $U_{\mathrm{w}}$ is positive and directed to the right (backward). $U_{\mathrm{f}}=U_{\mathrm{w}}-U$ for the frozen mode implies that when the value of $U$ (positive when directed forward) is subtracted from the value of $U_{\mathrm{w}}$ (positive when directed backward) to give a positive value for $U_{\mathrm{f}}$ then the frozen velocity is pointed backward. The lateral velocity is positive when directed upward and negative when directed downward (Fig. 3).

All forces considered in this work are parallel to the length of the plate/body and the fin. The thrust force on the fin is positive to the left (forward) while the drag force on the fin is positive to the right (backward). The resultant force on the fin (=thrust - drag) is positive to the left (forward). By action-reaction the thrust force on the fluid is positive to the right (backward) while the drag force on the fluid is positive to the left (forward). The resultant force on the fluid due to thrust and drag is positive to the right (backward).

Table 3 | Simulation parameters

\begin{tabular}{|c|c|c|c|c|}
\hline & Knifefish & Eel & Zebrafish (larval) & Mackerel \\
\hline Wavelength $\lambda(\mathbf{c m})$ & 3.75 & 1 & 0.31 & \\
\hline Frequency $f(\mathbf{H z})$ & 10.3 & 5 & 33.3 & 15.7 \\
\hline Fluid viscosity (Pa s) & $0.9 \times 10^{-3}$ & $1.4 \times 10^{-5}$ & $0.9 \times 10^{-3}$ & $0.9 \times 10^{-3}$ \\
\hline Wave velocity $(\mathrm{cm} / \mathrm{s})$ & 38.62 & 5 & 10.32 & 15.7 \\
\hline Translational velocity $(\mathrm{cm} / \mathrm{s})$ & 11.9 & 2.89 & 1.125 & 10 \\
\hline
\end{tabular}




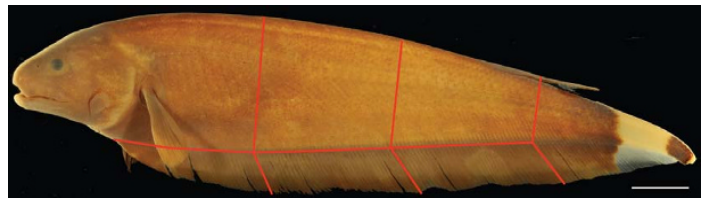

Figure $12 \mid$ The lines along which the fin length, fin height, and body height were measured for Apteronotus albifrons. Scale bar: $10 \mathrm{~mm}$. The photograph, ichthyology specimen 78148 (mczbase.mcz.harvard.edu/ guid/MCZ:Ich:78148), is reproduced with permission from the Museum of Comparative Zoology, Harvard University.

Measurements of fin and body size of South American weakly electric fishes. A group of 13 species in 8 genera in the family Apteronotidae of South American weakly electric fishes were considered. The family Apteronotidae is one of five families encompassing 32 genera and 135 species of South American knifefishes ${ }^{29}$. We restricted our measurements to a subset of one family for practical reasons, but it is evident from illustrations and images of knifefish in other families that the Apteronotidae are quite typical in terms of the body height to fin height issue examined here ${ }^{27,29,30}$.

An image of each specimen was provided by Andrew Williston of the Museum of Comparative Zoology, Harvard University. The fin and body height measurements were made at three locations along the fin length from the rostral tip: 25, 50, and 75 percent. The height of the fin was measured by measuring the length of the collapsed fin ray. The height of the body was measured along a line that was perpendicular to the body axis. The lines of measurement are shown in Fig. 12. The measured data are tabulated in Table 2. For specimens in which fin rays were not present for measurements at a needed position along the fin, ray length was estimated based on the trend of neighboring fin ray lengths. These data points are marked by an asterisk in the table.

1. Grillner, S. The motor infrastructure: from ion channels to neuronal networks. Nat. Rev. Neurosci. 4, 573-586 (2003).

2. McLean, D. L., Masino, M. A., Koh, I. Y., Lindquist, W. B. \& Fetcho, J. R. Continuous shifts in the active set of spinal interneurons during changes in locomotor speed. Nat. Neurosci. 11, 1419-1429 (2008).

3. Turner, R. W., Maler, L. \& Burrows, M. Special issue on electroreception and electrocommunication. J. Exp. Biol. 202, 1167-1458 (1999).

4. Krahe, R. \& Fortune, E. S. Electric fishes: neural systems, behaviour and evolution. J. Exp. Biol. 216, 2363-2364 (2013).

5. MacIver, M. A., Fontaine, E. \& Burdick, J. W. Designing future underwater vehicles: principles and mechanisms of the weakly electric fish. IEEE J. Oceanic Eng. 29, 651-659 (2004).

6. Neveln, I. D. et al. Biomimetic and bio-inspired robotics in electric fish research. J. Exp. Biol. 216, 2501-2514 (2013).

7. Colgate, J. E. \& Lynch, K. M. Mechanics and control of swimming: A review. IEEE J. Oceanic Eng. 29, 660-673 (2004).

8. Webb, P. W. Form and function in fish swimming. Sci. Am. 251, 58-68 (1984).

9. Lighthill, J. Mathematical biofluiddynamics (SIAM, Philadelphia, PA, 1975).

10. Lighthill, J. Large-amplitude elongated-body theory of fish locomotion. Proc. Roy. Soc. B. 179, 125-138 (1971).

11. Lighthill, J. \& Blake, R. Biofluiddynamics of balistiform and gymnotiform locomotion 1. Biological background, and analysis by elongated-body theory. J. Fluid Mech. 212, 183-207 (1990).

12. Blake, R. W. Swimming in the electric eels and knifefishes. Can. J. Zool. 61, 1432-1441 (1983).

13. Ruiz-Torres, R., Curet, O. M., Lauder, G. V. \& MacIver, M. A. Kinematics of the ribbon fin in hovering and swimming of the electric ghost knifefish. J. Exp. Biol. 216, 823-834 (2013).

14. Roper, M. \& Brenner, M. P. A nonperturbative approximation for the moderate reynolds number navierstokes equations. Proc. Natl. Acad. Sci. U.S.A 106, 2977-2982 (2009)

15. Shirgaonkar, A. A., Curet, O. M., Patankar, N. A. \& MacIver, M. A. The hydrodynamics of ribbon-fin propulsion during impulsive motion. J. Exp. Biol. 211, 3490-3503 (2008).

16. Neveln, I. D. et al. Undulating fins produce off-axis thrust and flow structures. J. Exp. Biol. 217, 201-213 (2014).
17. Curet, O. M., Patankar, N. A., Lauder, G. V. \& MacIver, M. A. Mechanical properties of a bio-inspired robotic knifefish with an undulatory propulsor. Bioinspir. Biomim. 6 (2011).

18. Videler, J. J. \& Hess, F. Fast continuous swimming of two pelagic predators, saithe (pollachius virens) and mackerel (scomber scombrus): A kinematic analysis. J. Exp. Biol. 109, 209-228 (1984).

19. Tytell, E. D. \& Lauder, G. V. The hydrodynamics of eel swimming: I. Wake structure. J. Exp. Biol. 207, 1825-1841 (2004).

20. Borazjani, I. \& Sotiropoulos, F. Numerical investigation of the hydrodynamics of carangiform swimming in the transitional and inertial flow regimes. J. Exp. Biol. 211, 1541-1558 (2008)

21. MacIver, M. A., Sharabash, N. M. \& Nelson, M. E. Prey-capture behavior in gymnotid electric fish: Motion analysis and effects of water conductivity. J. Exp. Biol. 204, 543-557 (2001)

22. Shirgaonkar, A. A., MacIver, M. A. \& Patankar, N. A. A new mathematical formulation and fast algorithm for fully resolved simulation of self-propulsion. J. Comput. Phys. 228, 2366-2390 (2009).

23. Peng, J. \& Dabiri, J. The 'upstream wake' of swimming and flying animals and its correlation with propulsive efficiency. J. Exp. Biol. 211, 2669-2677 (2008).

24. Fish, F. E. \& Lauder, G. V. Passive and active flow control by swimming fishes and mammals. Ann. Rev. Fluid Mech. 38, 193-224 (2006).

25. Schultz, W. W. \& Webb, P. W. Power requirements of swimming: Do new methods resolve old questions? Integr. Comp. Biol. 42, 1018-1025 (2002).

26. Shen, L., Zhang, X., Yue, D. K. P. \& Triantafyllou, M. S. Turbulent flow over a flexible wall undergoing a streamwise travelling wave motion. J. Fluid Mech. 484, 197-221 (2003)

27. Albert, J. S. Species diversity and phylogenetic systematics of american knifefishes (gymnotiformes, teleostei). Misc. Publ. Mus. Zool. Univ. Mich. 190, 1-127 (2001).

28. Kern, S. \& Koumoutsakos, P. Simulations of optimized anguilliform swimming. J. Exp. Biol. 209, 4841-4857 (2006).

29. Albert, J. S. \& Crampton, W. G. R. Diversity and phylogeny of neotropical electric fishes (Gymnotiformes). In Electroreception, 360-409 (Springer, New York, 2005).

30. Crampton, W. G. R. \& Albert, J. S. Evolution of electric signal diversity in gymnotiform fishes. In Ladich, F., Collin, S., Moller, P. \& Kapoor, B. (eds.) Communication in Fishes, 641-725 (Science Publishers Inc., Enfield, NH, 2006).

\section{Acknowledgments}

This work was supported by NSF grants CBET-0828749 to N.A.P. and M.A.M, CMMI-0941674 to M.A.M. and N.A.P, and CBET-1066575 to N.A.P. Computational resources were provided by NSF's TeraGrid Project grants CTS-070056T and CTS-090006, and by Northwestern University High Performance Computing System - Quest.

\section{Author contributions}

N.A.P., A.A.S., R.B., M.A.M. and I.D.N. conceived and designed the research and experiments. R.B. and A.A.S. performed numerical simulations. I.D.N. and R.B. performed experiments. N.A.P., R.B., A.A.S. and M.A.M. analyzed data. A.A.S., A.P.S.B. and N.A.P. contributed the numerical simulation tool. I.D.N. and M.A.M. contributed the experimental tool. R.B., A.A.S., N.A.P. and M.A.M. wrote the paper. All authors edited the paper. R.B. and A.A.S. made equal contribution.

\section{Additional information}

Supplementary information accompanies this paper at http://www.nature.com/ scientificreports

Competing financial interests: The authors declare no competing financial interests.

How to cite this article: Bale, R. et al. Separability of drag and thrust in undulatory animals and machines. Sci. Rep. 4, 7329; DOI:10.1038/srep07329 (2014).

This work is licensed under a Creative Commons Attribution-NonCommercialShareAlike 4.0 International License. The images or other third party material in this article are included in the article's Creative Commons license, unless indicated otherwise in the credit line; if the material is not included under the Creative Commons license, users will need to obtain permission from the license holder in order to reproduce the material. To view a copy of this license, visit http:// creativecommons.org/licenses/by-nc-sa/4.0/ 